\title{
Quantification of Ammonium Sulphate Nitrate (ASN) fertilizers
}

\author{
J. M. Montejo-Bernardo ${ }^{1}$, S. García-Granda ${ }^{1, *}$, \\ A. Fernández-González² \\ ${ }^{1}$ SYSTAM Group, X-ray Team, Department of Physical and Analytical Chemistry, Faculty \\ of Chemistry, University of Oviedo-CINN, Spain \\ ${ }^{2}$ Molecular Recognition Team, Department of Physical and Analytical Chemistry, Faculty of \\ Chemistry, University of Oviedo-CINN, Spain \\ *Contact author; e-mail: sgg@uniovi.es
}

Keywords: ASN fertilizers, quantification, X-ray powder diffraction

\begin{abstract}
This paper shows a simple procedure for the quantification of industrial and laboratory samples of Ammonium Sulphate-Nitrate fertilizers (ASN) based on a Rietveld fit from $\mathrm{X}$-ray powder diffraction profiles. The Rietveld fit is performed by means of the structural models of the double salts $2 \mathrm{NH}_{4} \mathrm{NO}_{3} \cdot\left(\mathrm{NH}_{4}\right)_{2} \mathrm{SO}_{4}$ and $3 \mathrm{NH}_{4} \mathrm{NO}_{3} \cdot\left(\mathrm{NH}_{4}\right)_{2} \mathrm{SO}_{4}$, previously reported by the authors. The proposed method demonstrated to be highly accurate even when medium-low quality X-ray Powder Diffraction profiles are used. The result of the composition of $\mathrm{H}, \mathrm{N}$ and $\mathrm{S}$ in the samples is very similar to those obtained by elemental analysis.
\end{abstract}

\section{Introduction}

X-ray Powder Diffraction techniques are mainly used as identification tools in the fertilizer field [1-4], leaving the quantification as an unexploited field. This is mainly due to two reasons, i) the co-existence in the sample of minority phases which may be unknown or difficult to quantify and ii) a lack of data about the structure of some of the phases present in the sample. Till now, XRPD analysis used to require sample pre-treatment, internal standards or post-analysis data processing leading to an increase in the error. As far as we know, the current state-of-the-art in this field covers semiquantitative analysis of phosphonate fertilizers using an internal standard to obtain an accuracy in the 10-15\% absolute range for the major components [5-7], quantitative analysis using mixtures of different internal standards yielding relative errors around 10\% [8-9], comparative methods based on the comparison of the intensities of every phase in the fertilizer with the profiles of the pure components, resulting in absolute errors around 2\% [10] and methods using as internal standard either alumina in phosphonate or nitrate samples [11] or spinel in potassium silicate fertilizers with a high degree of uncertainty (20\%) [12]. Quantification of ASN fertilizers has been, however, poorly studied. Highsmith et al. have reported a quantification procedure with about 5\% absolute errors and 2\% reproducibility [13], based on the Matrix Flushing [Normalized RIR] Method [14]. 
Crystal structures of the double salts $2 \mathrm{NH}_{4} \mathrm{NO}_{3} \cdot\left(\mathrm{NH}_{4}\right)_{2} \mathrm{SO}_{4}$ and $3 \mathrm{NH}_{4} \mathrm{NO}_{3} \cdot\left(\mathrm{NH}_{4}\right)_{2} \mathrm{SO}_{4}$, which are described to be together with ammonium sulphate the main components of ASN fertilizers, have been previously reported by the authors [15]. This structural characterization now allows the quantification of industrial samples of ANS fertilizers using XRPD and a Rietveld fit of the experimental data [16-18], as described in this work. Validation of the method is shown here for several real samples, including some with poor profiles (broad and undefined peaks, showing important overlap). The results of the composition of $\mathrm{H}, \mathrm{N}$ and $\mathrm{S}$ in the samples were compared with those obtained by elemental analysis.

\section{Samples and experimental data}

Samples $\mathbf{1}$ and $\mathbf{4}$ were provided by the company Fertiberia S.A, whereas samples $\mathbf{2}$ and $\mathbf{3}$ were prepared at the laboratory..

Experimental XRPD patterns were acquired in transmission mode on an Agilent Xcalibur diffractometer NOVA at the X-ray Diffraction Unit of the Scientific-Technological Services of the University of Oviedo for samples $\mathbf{1}$ and $\mathbf{3}$, and on a Gemini $\mathrm{S}$ at the X-ray Group laboratory for samples 2 and $\mathbf{4}$, using Cu Ka radiation $(\lambda=1.5418 \AA)$, with an Onyx CCD area detector $(165 \mathrm{~mm})$ and a Ruby CCD area detector $(135 \mathrm{~mm})$, respectively. The powder patterns were collected using three $2 \theta$ detector positions $-82,0^{\circ}$ and $+82^{\circ}$. For each detector position the sample was rotated $300^{\circ}$ on $\varphi$, with $300 \mathrm{~s}$ for each rotation, and finally the integrated data was averaged on the $2 \theta$ range $3-60^{\circ}$ for sample $\mathbf{1}$ and $3,3-55^{\circ}$ for sample 2 , and $5-50^{\circ}$ for sample 4. Peak intensities were integrated over the entire ring in step-scan mode, using a step size of $0.02^{\circ}$ for samples $\mathbf{1}, \mathbf{3}$ and $\mathbf{4}$, and $0.03^{\circ}$ for sample 2 . Rietveld refinements were performed in the range $5-50^{\circ}$ for every sample via the FULLPROF program package [19] using a pseudo-Voigt peak-shape function for samples $\mathbf{1}, \mathbf{4}$, and a Pearson VII function for sample $\mathbf{2}$ and $\mathbf{3}$. In least-squares cycles, the refined parameters were: zero-shift error, background coefficients (six-coefficients polynomial function), unit-cell parameters, peakshape parameters ( $\eta$ for pseudo-Voigt and $m$ for Pearson VII), phase linewidths (from the Caglioti formula: $\mathrm{U}, \mathrm{V}$ and $\mathrm{W}$ for $\mathbf{1}$, and $\mathrm{W}$ for $\mathbf{2 ,} 3$ and $\mathbf{4}$ ), an asymmetry parameter, and scale factor. Atoms were modeled as anisotropic. Profile plots were obtained using WinPLOTR [20].

Elemental analyses were carried out in a Perkin-Elmer 2400 Elemental Analyser at the Unit of Elemental Analysis and Thermocalorimetry of the Scientific-Technological Services of the University of Oviedo. Validity of the results was contrasted with a sample of known composition (Ammonium Sulphate).

\section{Results and discussion}

\subsection{Percentage composition of AS patron sample}

Theoretical and estimated composition of the Ammonium Sulphate sample are shown at the top of table 1. Values of $\mathrm{H}, \mathrm{N}$ and $\mathrm{S}$ are obtained as the mean of two measurements whereas oxygen is calculated by subtraction. As it can be seen, hydrogen is approximately $10 \%$ underestimated while sulphur appears with about $5 \%$ of overestimation. Oxygen appears underestimated as well. Such deviations must be taken into account when comparing the percentages of $\mathrm{H}, \mathrm{N}$ and $\mathrm{S}$ coming from elemental analysis with those obtained from the phase composition calculated by Rietveld fit. 


\subsection{Sample 1. Industrial fertilizer}

According to the composition provided by the manufacturing factory Fertiberia, the sample is $60.8 \% \mathrm{AS}, 37.7 \% \mathrm{AN}(\mathrm{w} / \mathrm{w})$ and $1.5 \%$ minority phases (such as Fe or anti-caking stabilizers). Figure 1 shows the Rietveld fit for this sample as well as the weight percentages and the $R_{F}$ and $R_{B}$ parameters for each of the three analysed phases. From our results, the sample contains $57.6 \%$ AS and $40.9 \%$ AN, quite close to the expected values. Estimated percentage composition and the values obtained by elemental analysis are shown in table 1 .

\subsection{Sample 2. Double salt 3·1}

This sample was prepared and crystallized in our laboratory according to the described procedure in [21]. This sample contains $4.9 \%$ of free AN [18] as the average of three aliquots. figure 2 shows the Rietveld fit of one of the measurements as well as the weight percentages and the $\mathrm{R}_{\mathrm{F}}$ and $\mathrm{R}_{\mathrm{B}}$ parameters for every one of the two phases. The phase composition is very close to theoretical values, and percentages of $\mathrm{H}, \mathrm{N}, \mathrm{O}$ and $\mathrm{S}$ are very similar that the corresponding values obtained by elemental analysis (table 1 ).

\subsection{Sample 3. Mixture}

This sample was prepared in our laboratory to be $80.4 \%$ 3AN·AS, $15.5 \%$ free AS and $4.1 \%$ free AN. Percentages from Rietveld fit and the values of the $R_{F}$ and $R_{B}$ parameters for every phase are shown in figure 3 , with differences in percentages lower than $2 \%$. Once more, the results of the elemental analysis are close that calculated from the Rietveld fit (table 1). This confirms that both techniques point very close to expected values.

\subsection{Sample 4. Industrial fertilizer}

Provided by Fertiberia as an unknown sample. Low values of $R_{F}$ and $R_{B}$ indicate a good Rietveld fit, and the elemental composition data derived from the phase percentages are in good agreement with those arising from the HNS analysis (in the bottom of table 1).

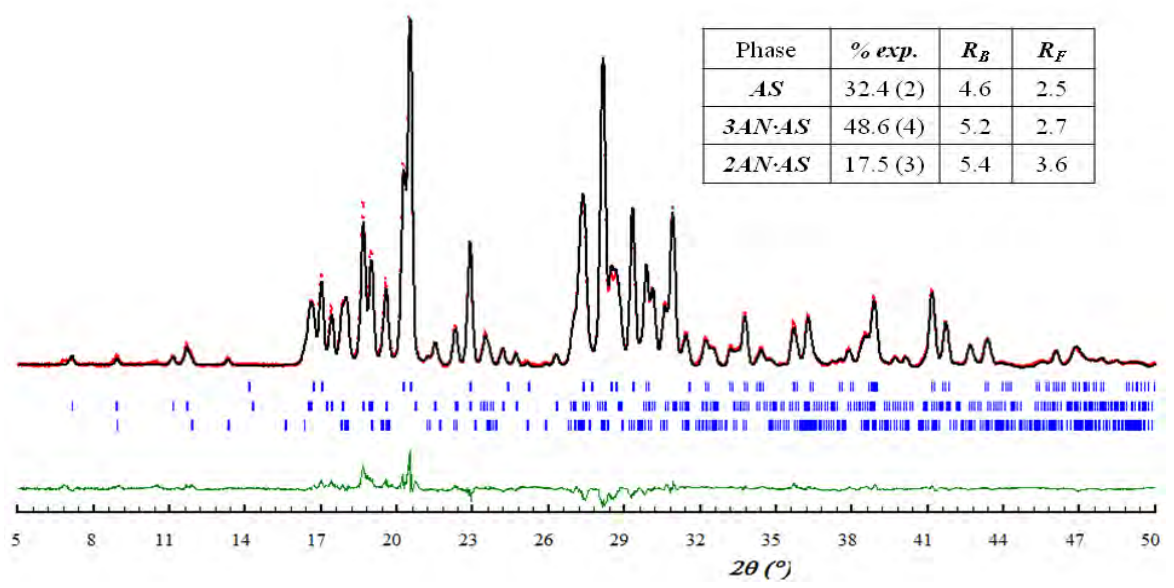

Figure 1. Rietveld refinement for sample 1, an industrial fertilizer. 


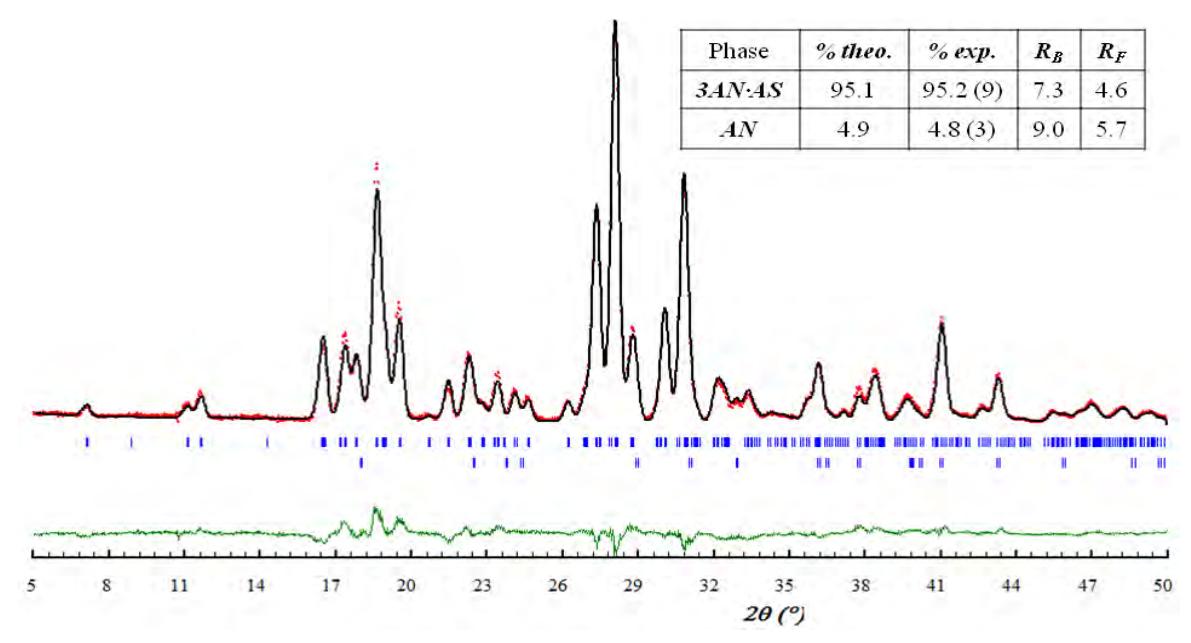

Figure 2. Rietveld refinement for sample 2, a double salt of AN and AS, with free AN.

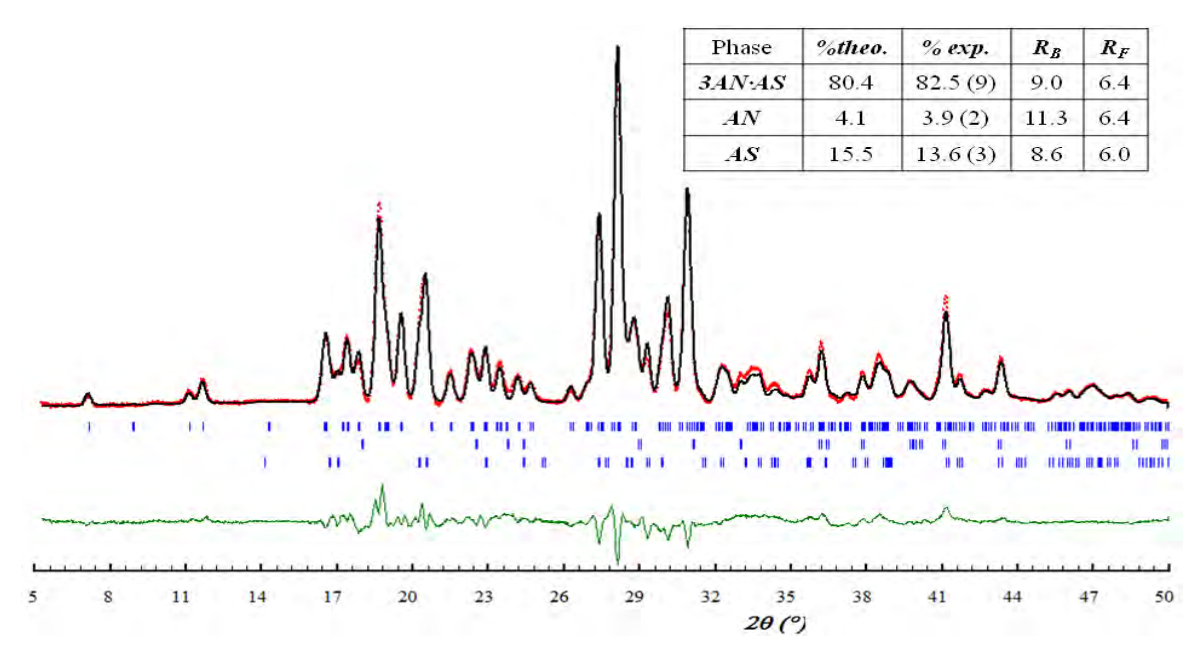

Figure 3. Rietveld refinement for sample 3, a mixture prepared in laboratory. 


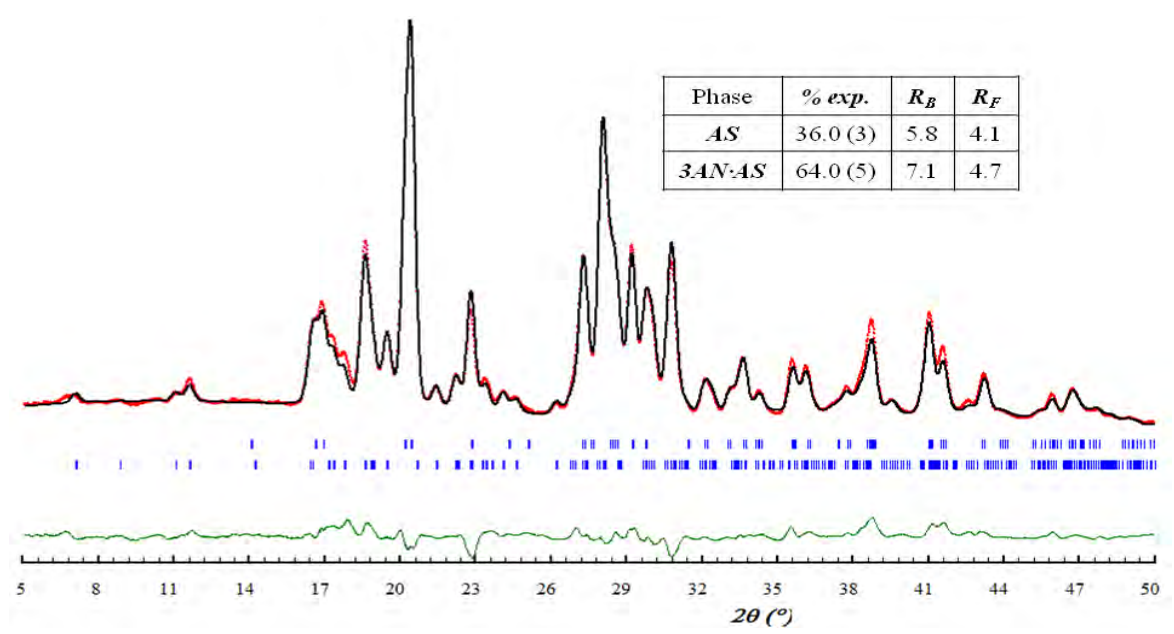

Figure 4. Rietveld refinement for sample 4, an industrial fertilizer.

Table 1. Comparison between the elemental composition obtained from phase composition from Rietveld fit (figures 1-4) and from NHS analysis.

\begin{tabular}{|c|c|c|c|c|c|}
\hline & Elements(\%) & $\boldsymbol{H}$ & $\boldsymbol{N}$ & $\boldsymbol{O}$ & $\boldsymbol{S}$ \\
\hline \multirow{3}{*}{ AS sample } & Theoretical & $\mathbf{6 . 1 0}$ & $\mathbf{2 1 . 2 0}$ & $\mathbf{4 8 . 4 3}$ & $\mathbf{2 4 . 2 7}$ \\
\cline { 2 - 6 } & HNS analysis & 5.40 & 21.62 & 47.38 & 25.60 \\
\hline \multirow{3}{*}{ Sample 1 } & $\boldsymbol{X}$-ray analysis & $\mathbf{5 . 5 8}$ & $\mathbf{2 6 . 5 3}$ & $\mathbf{5 2 . 4 2}$ & $\mathbf{1 3 . 9 7}$ \\
\cline { 2 - 6 } & HNS analysis & 5.18 & 25.48 & 52.48 & 15.36 \\
\hline \multirow{3}{*}{ Sample 2 } & $\boldsymbol{X}$-ray analysis & $\mathbf{5 . 4 0}$ & $\mathbf{3 0 . 3 4}$ & $\mathbf{5 6 . 0 7}$ & $\mathbf{8 . 1 9}$ \\
\cline { 2 - 6 } & HNS analysis & 4.20 & 31.78 & 53.83 & 10.19 \\
\hline \multirow{3}{*}{ Sample 3 } & $\boldsymbol{X}$-ray analysis & $\mathbf{5 . 5 0}$ & $\mathbf{2 9 . 0 8}$ & 55.02 & $\mathbf{1 0 . 4 0}$ \\
\cline { 2 - 6 } & HNS analysis & 4.99 & 30.44 & 51.46 & 13.11 \\
\hline \multirow{2}{*}{ Sample 4 } & $\boldsymbol{X}$-ray analysis & 5.66 & 26.90 & 53.19 & 14.25 \\
\cline { 2 - 6 } & HNS analysis & 5.01 & 26.98 & 51.91 & 16.10 \\
\hline
\end{tabular}

\section{Concluding remarks}

Samples of fertilizers, either with industrial or laboratory origin, have been accurately quantified by XRPD Rietveld fits (including samples with poor profiles). The good results obtained were checked with HNS analysis. In every case, the compositions obtained through Rietveld fit and from elemental analysis are in good agreement. 


\section{References}

1. Coates, R.V. \& Woodard, G.D., 1963, J. Sci. Food Agric., 14, 398.

2. Malquori, A. \& Cecconi, S., 1964, Chim. Ind., 46, 637.

3. Lehr, J.R., Brown, E.H., Frazier, A.W., Smith, J.P. \& Thrasher, R.D, 1967, Chem. Eng. Bull., 6, 166.

4. Jassal, H.S. \& Kumar, R, 2004, Fertilizer News, 49, 35.

5. Ando, J. \& Akiyama, T, 1964, Bunseki Kagaku, 13, 717.

6. Ando, J., Smith, J.P., Siegel, M.R. \& Jordan, J.E, 1965, J. Agric. Food Chem., 13, 186.

7. Cekinski, E, 1987, Fertilizantes, 9, 10.

8. Maslennikov, B.M. \& Soklakov, A.I, 1970, Rentgenogr. Mineralnogo Syrya, 7, 144.

9. Knob, B., Wankova, J., Moudry, F. \& Kuba, M, 1986, Powder Diffr., 1, 40.

10. Vonk, C.G., Heck, H.G. \& Pijpers, A.P, 1977, in New Dev. Phosphate Fert. technol., Proc. Tech. Conf. ISMA Ltd, edited by L.J. Carpentier (Elsevier) pp. 115.

11. Akiyama, T, 1978, Nippon Dojo Hiryogaku Zasshi, 49, (5), 418.

12. Ando, J., Izumi, K., Ozawa, N. \& Esaki, M, 1995, Nip. Dojo Hiru. Zasshi, 66, (1), 13.

13. Highsmith, R.E., Kweeder, J.A. \& Correale, S.T, 2002, US Patent 0095966A1.

14. Snyder, R.L, 1992, Powder Diffr., 7, 186.

15. Montejo-Bernardo, J.M., García-Granda, S. \& Fernández-González, A, 2010, Acta Crystallogr., B66, 358.

16. Rietveld, H.M, 1967, Acta Crystallogr., 22, 151.

17. Rietvled, H.M, 1969, J. Appl. Crystallogr., 2, 65.

18. Hill, R.J., Howard, C.J., 1987, J. Appl. Crystallogr., 20, 467.

19. Rodriguez-Carvajal, J, 1990, FULLPROF. Abstracts of the Satellite Meeting on Powder Diffraction of the XV Congress of the IUCr, p.127, Toulouse, France.

20. Roisnel, T. \& Rodriguez-Carvajal, J, 2000, Mater. Sci. Forum, 378-381, 118.

21. Smith, J.P., Lehr, J.R. \& Frazier, A.W, 1962, J. Agric. Food Chem., 10, 77.

Acknowledgements. The authors gratefully acknowledge Fertiberia S.A. for supplying samples 1 and 4, FEDER funding, MICINN Projects MEC-06-MAT-2006-01997, MAT201015094, MEC-06-CTQ2006-14644-C02-01 and Factoría Española de Cristalización CSD2006-00015 for financial support. Our thanks also to Laura Roces and Beatriz Ramajo (SCTs) for the X-ray and elemental analysis measurements respectively. 